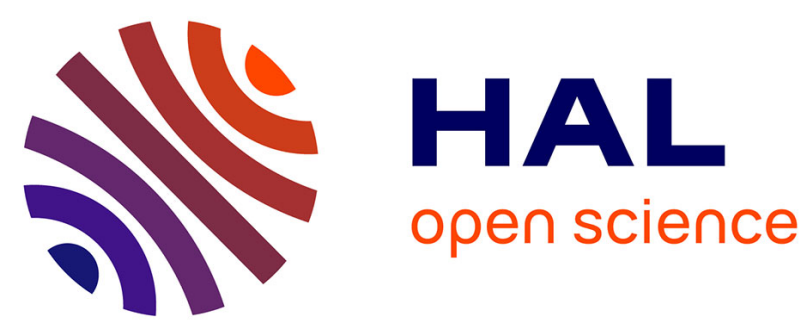

\title{
Investigation on cone jetting regimes of liquid droplets subjected to pyroelectric fields induced by laser blasts
}

Oriella Gennari, Luigi Battista, Benjamin Silva, Simonetta Grilli, Lisa Miccio,

Veronica Vespini, Sara Coppola, Pierangelo Orlando, Laurent Aprin, Pierre Slangen, et al.

\section{To cite this version:}

Oriella Gennari, Luigi Battista, Benjamin Silva, Simonetta Grilli, Lisa Miccio, et al.. Investigation on cone jetting regimes of liquid droplets subjected to pyroelectric fields induced by laser blasts. Applied Physics Letters, 2015, 106 (5), pp.054103. 10.1063/1.4907005 . hal-02011840

\author{
HAL Id: hal-02011840 \\ https://hal.science/hal-02011840
}

Submitted on 4 Feb 2020

HAL is a multi-disciplinary open access archive for the deposit and dissemination of scientific research documents, whether they are published or not. The documents may come from teaching and research institutions in France or abroad, or from public or private research centers.
L'archive ouverte pluridisciplinaire HAL, est destinée au dépôt et à la diffusion de documents scientifiques de niveau recherche, publiés ou non, émanant des établissements d'enseignement et de recherche français ou étrangers, des laboratoires publics ou privés. 


\title{
Investigation on cone jetting regimes of liquid droplets subjected to pyroelectric fields induced by laser blasts
}

\author{
Oriella Gennari, ${ }^{1, a)}$ Luigi Battista, ${ }_{1,}^{1, a)}$ Benjamin Silva, ${ }^{2}$ Simonetta Grilli, ${ }^{1, b}$ Lisa Miccio, ${ }^{1}$ \\ Veronica Vespini, ${ }^{1}$ Sara Coppola, ${ }^{1}$ Pierangelo Orlando, ${ }^{3}$ Laurent Aprin, ${ }^{2}$ Pierre Slangen, ${ }^{2}$ \\ and Pietro Ferraro ${ }^{1}$ \\ CNR Istituto di Cibernetica, Via Campi Flegrei 34, 80078 Pozzuoli (NA), Italy \\ Ecole des mines d'Alés, 30100 Alés, France \\ CNR Istituto di Biochimica delle Proteine, Via Campi Flegr
}

\begin{abstract}
Electrical conductivity and viscosity play a major role in the tip jetting behaviour of liquids subjected to electrohydrodynamic (EHD) forces, thus influencing significantly the printing performance. Recently, we developed a nozzle- and electrode-free pyro-EHD system as a versatile alternative to conventional EHD configurations and we demonstrated different applications, including inkjet printing and three-dimensional lithography. However, only dielectric fluids have been used in all of those applications. Here, we present an experimental characterization of the pyro-EHD jetting regimes, induced by laser blasts, of sessile drops in case of dielectric and conductive liquids in order to extend the applicability of the system to a wider variety of fields including biochemistry and biotechnology where conductive aqueous solutions are typically used.
\end{abstract}

The electrohydrodynamic (EHD) jetting in which liquids emerge from capillary tubes, nozzles, or orifices is used in a number of technological fields including spray coating, ${ }^{1}$ inkjet printing, ${ }^{2-6}$ patterning of biological suspensions, ${ }^{7-9}$ spraying of agricultural chemicals, ${ }^{10}$ mass spectrometry, ${ }^{11}$ and film deposition. ${ }^{12}$ Electric fields play an important role also in processes of natural occurrence such as the formation of raindrops in thunderclouds ${ }^{13}$ and nuclear physics. ${ }^{14}$ The way the liquid droplets are formed under electric fields is of fundamental importance in all of these applications. The early works from Taylor and McEwan on the stability of a horizontal fluid interface stimulated by a vertical electric field, are worthy of mention. ${ }^{15,16}$ Thereafter, a huge amount of both theoretical and experimental studies, focused on different configurations, have been performed and have helped to elucidate the basic mechanisms. These include some early works from Basaran's group,${ }^{17-20}$ and from Hayati et al. who studied the mechanism of EHD spraying. ${ }^{21}$ More recent works deal with other specific topics such as the effects of bulk charges and relaxation time on arc electrospraying, ${ }^{22}$ the scaling laws for the disintegration of electrified drops, ${ }^{23}$ the characterization and optimization of experimental parameters in jetting performance ${ }^{24-27}$ the oscillations of water droplets on insulating surfaces, ${ }^{28}$ the EHD response of low-conductivity pendant drops to a step change in the electric field ${ }^{29}$ the liquid meniscus oscillations and tip streaming under continuous and pulsed voltages, ${ }^{30-32}$ the development of a multi-nozzle EHD device ${ }^{33}$ the investigation on the regimes of water-oil interface, ${ }^{34}$ numerical simulation of cone-jet formation in electrostatic spray, ${ }^{35}$ and the stability of liquid bridges between two opposite Taylor cones. ${ }^{36}$

\footnotetext{
a) The authors L. Battista and O. Gennari contributed equally to this work

${ }^{b)}$ Author to whom correspondence should be addressed. Electronic mail: simonetta.grilli@cnr.it.
}

However, the most studied configuration is that used in EHD inkjet devices where an electric field is established between a source nozzle and an extractor electrode. An electric charge is induced at the surface of the liquid meniscus, creating an electric stress that stretches the meniscus in the direction of the field, and a droplet or jet of liquid is formed when the electrostatic force is stronger than the surface tension. An EHD inkjet head can produce droplets smaller than the size of the nozzle. This unique feature distinguishes EHD printing from conventional methods by allowing sub-micron resolution. $^{2}$

Only a few works deal with the shape evolution of sessile drops ${ }^{17,30,37}$ or liquid layers ${ }^{38}$ immersed in ambient air under the action of an electric field. Anyway, these works make use of external electrodes, and no attention at all has been devoted to the behaviour of sessile drops immersed in ambient air and subjected to pyroelectric charges in case of low and high conductivity. Recently, we developed a pyro-EHD printing system free from nozzles and electrodes and based on the use of surface charges generated spontaneously onto pyroelectric crystals. ${ }^{39}$ That work demonstrated the capability of the system for fine printing of oil-based inks and discussed deeply the advantages compared to well-established EHD printing heads. Among these assets the nozzle-free condition allows one to avoid cross-contaminations and detrimental cloggings of the orifice. Use of spontaneous charges allows one to circumvent high voltage external electrodes and circuits and can facilitate the parallelization of the process. Nowadays, the pyro-EHD system has been demonstrated useful for diverse applications that make use only of dielectric fluids, ${ }^{40-43}$ and it is well known that the conductivity of the fluid influences significantly the EHD behaviour. Therefore, we believe that it is of great importance to study experimentally the behaviour of sessile drops of dielectric and conductive liquids under the action of pyroelectric fields, in order to identify the working 
regimes and so to extend the applicability of this promising inkjet printing technology to conductive fluids. This would enable the pyro-EHD to find application also in fields like biochemistry and biotechnology where typically one deals with high-conductive aqueous solutions.

Here, we report the experimental investigation of the cone jetting regimes of sessile drops of dielectric and conductive liquids, subjected to pyroelectric fields induced by laser shoots. The early stages of the cone formation and the successive temporal evolution were visualized and evaluated by a high-speed camera and were characterized in terms of different parameters, including shape and temporal evolution. Figure 4(a) in Ref. 39 shows the schematic view of the pyro-EHD dispenser and details about the principle of operation can be found therein. Basically, a base drop of $0.5 \mu \mathrm{L}$ was deposited by standard pipette onto a hydrophobic substrate (silicone rubber). A standard glass coverslip was mounted parallel on the top and represented the target slide where depositing the working fluid. The key component responsible for the generation of the pyroelectric field was a wafer of $\mathrm{LiTiO}_{3}$ (LT, z-cut, $0.5 \mathrm{~mm}$ thick) mounted over the target slide. The beam exiting a $\mathrm{CW} \mathrm{CO}_{2}$ laser (ULR-10, wavelength $10.6 \mu \mathrm{m}$, output optical power $10 \mathrm{~W}$, and beam size around $4 \mathrm{~mm}$ ) impinged directly onto the LT wafer, in correspondence of the base drop, thus enabling the generation of pyroelectric surface charges on the crystal (around $10^{-8}$ coulomb $^{44}$ ). The output of the $\mathrm{CO}_{2}$ laser was switchable on and off by a $5 \mathrm{~V}$ signal delivered via a TTL port, and typically, the crystal was stimulated by applying manually one $5 \mathrm{~V}$ square pulse, through the trigger of a conventional signal generator.

According to the well-known principles of EHDs, the pyroelectric field, generated by the laser stimulation, induced the base drop to evolve under the action of electric, capillary, and viscous forces. Here we focus the attention on the role of the electrical conductivity of the working fluid in the EHD behaviour of a sessile drop subjected to a pyroelectric field. Two classes of liquid samples with common conductive and dielectric properties were used. The conductive liquid (CL) group included tap water, distilled water, water suspension of polystyrene beads (diameter $2 \mu \mathrm{m}$, Sigma Aldrich L4530), and a solution of biomolecules. The dielectric liquid (DL) group included almond, soy, and mineral oil. In particular, the biomolecule solution consisted of a short oligonucleotide (15-20 bp) solubilized in Tris-EDTA (TE) buffer solution and mixed with an advanced buffer system (1:1 ratio) (Micro-Spotting Solution Plus $2 \times$, ArrayIt). A high-speed CMOS camera (Motion Pro Y3-S1, pixel size $10.85 \times 10.85 \mu \mathrm{m}$ ) was used for acquiring the side view of the drop during the experiments. The camera provided a frame rate up to around $25000 \mathrm{fps}$ with a spatial resolution of $1280 \times 128$ pixel and up to $88000 \mathrm{fps}$ with a spatial resolution of $1280 \times 16$ pixel. The same voltage generator was used for triggering simultaneously the $\mathrm{CO}_{2}$ laser and the camera and the image sequence was around $2 \mathrm{~s}$ long for each experiment. The crystal was stimulated by one $\mathrm{CO}_{2}$ emission $250 \mathrm{~ms}$ long in each experiment.

The supplementary movies ${ }^{45} 1$ and 2 show the typical evolution of the drop profile under the pyro-EHD stimulation for a CL and for a DL, respectively. The movies were acquired at $12100 \mathrm{fps}$ with a ROI of $(296 \times 1280)$ pixel, ${ }^{2}$ but are shown in slow motion for better visualization. The two liquid families exhibited clearly two different regimes. The CLs exhibited a regime that we call here "pulsating," due to fast cone formation and fast restoring of the equilibrium condition just after the cone formation. In fact, the average life of the cone was around $0.1 \mathrm{~ms}$ and a typical oscillating behaviour of the drop from sharp cone to the equilibrium profile was established. Moreover, in case of the CLs, the meniscus profile exhibited a typical "conic cusping singularity," in agreement with previous studies performed by traditional electrode-based EHD. ${ }^{38}$ Conversely, the DLs exhibited a regime that we call here "steady," due to the slower growth of the conical meniscus and to the persistence of the cone profile during the tip jetting process that evolves into a dripping-like mode. The equilibrium profile was restored only by reducing and ultimately removing the thermal gradient, namely, the pyroelectric field. Moreover, the meniscus profile of the DLs showed a sharper protrusion shape, again in agreement with Ref. 38. Figures 1(a) and 1(b) show the snapshots of the meniscus profile observed typically in case of CLs (a) and DLs (b). Figures 1(c) and 1(d) show the typical temporal evolution of the meniscus profile in case of the oligonucleotide solution (CL group) and of the almond oil (DL group), respectively. The figures were obtained by overlapping the snapshots acquired during the cone formation. The formation of the protrusion and cusp shapes is clearly visible for the two liquid families. Figure 2(a) shows the temporal variation of the drop height till the formation of the cone, for all of the samples. The CLs exhibit faster cone formation and shorter cone heights, in agreement with previous theoretical interpretations. ${ }^{38}$ Figure 2(b) shows the distribution of the time formation of the cone for all of the samples. It is clearly visible that the two families have two time ranges, with the CLs showing a time for cone formation that, in average, is around 5 times shorter than that observed for the DLs. In case of CLs, a fast oscillation of the meniscus profile was observed prior to cone jetting, and Fig. 2(c) shows the typical temporal variation of the height of the drop apex during these oscillations. The heights were evaluated from the snapshots of a movie acquired at $12100 \mathrm{fps}$ and denote an oscillation frequency around $300 \mathrm{~Hz}$, namely, the same order of magnitude predicted in Ref. 46. Although the viscosity of the surrounding medium is much lower here compared to Ref. 46, the same order of magnitude value may be attributed to the impulsive and transient nature of the field generated pyroelectrically, compared to that generated by a standard source.

Figure 3(a) reports also the evaluation of the cone semiangle (by applying the software NIH ImageJ) for each liquid studied in this work. The CL group exhibited semi-angles in the range of $48^{\circ}$ while the typical cone semi-angle measured in case of the DLs was around $32^{\circ}$ in average, in agreement with theoretical and experimental studies reported in literature in case of standard EHD configurations. ${ }^{37}$

Figures 3(c) and 3(d) show schematically the side view of the fluid configuration considered in this work. An axisymmetric drop of incompressible viscous liquid and volume $V$ is attached onto an insulating plate. With gravity effects neglected, the stationary shape of the drop is a segment of 

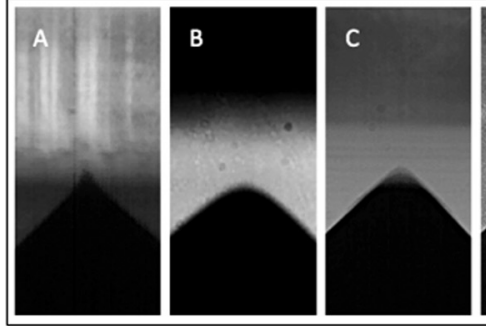

(a)

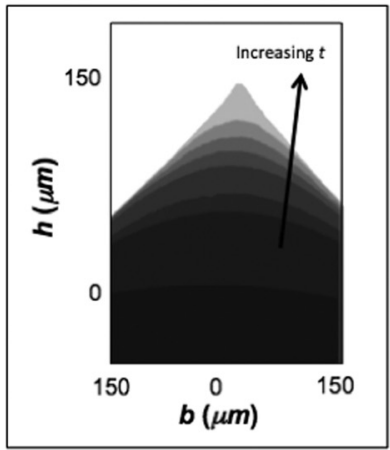

(c)

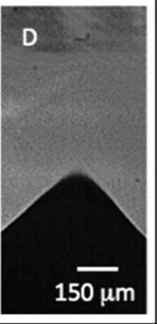
政
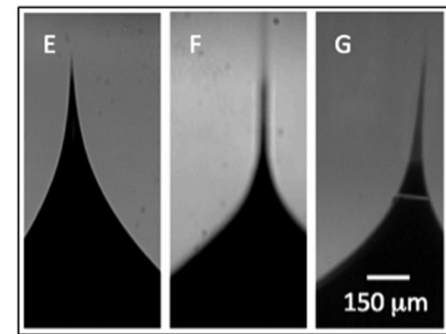

(b)

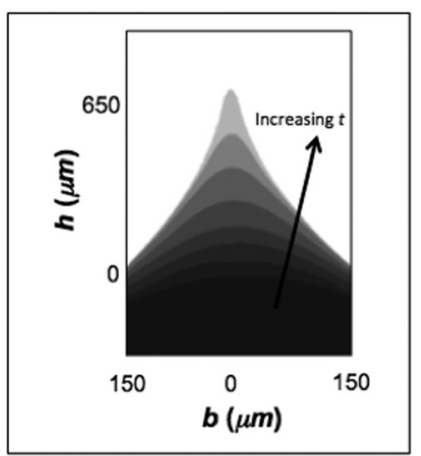

(d)
FIG. 1. Typical meniscus profiles of the base drop in case of different samples. The group in (a) refers to the CLs: A, distilled water; B, tap water; $\mathrm{C}$, oligonucleotide solution; $\mathrm{D}$, dispersion of PS beads. The group in (b) refers to the DLs: E, almond oil; F, mineral oil; and G, soy oil. Temporal evolution of the meniscus profile in case of two typical cases: (c) almond oil and (d) oligonucleotide solution. sphere with a static contact angle $\theta_{Y}$ defined by the mechanical equilibrium of three interfacial tensions, according to the well known Young's equation $\gamma_{l v} \cos \theta_{Y}=\gamma_{s v}-\gamma_{s l}$, where $\gamma_{l v}, \gamma_{s v}$, and $\gamma_{s l}$ represent the liquid-vapor, solid-vapor, and solid-liquid interfacial tensions, respectively (see Fig. 3(c)). The surrounding air is supposed to be an electric insulator. Figure 3(b) reports the experimental evaluation of the contact angles of the test liquids used in this work. The measurements of the contact angles were obtained from the snapshots of the resting drop, by applying the software NIH ImageJ. Once the pyroelectric effect is activated, an electric field surrounds the drop that therefore evolves under the action of the electric, capillary and viscous forces, as shown schematically in Fig. 3(d). While the potential increases, the drop deforms due to the accumulation of charges on the surface. The fluid is accelerated continuously in what is a nearly spherically symmetric flow and assumes the Taylor cone profile, which is maintained over a certain critical limit of the electric potential. As shown in the seminal work of Taylor, ${ }^{15}$ once exceeded that limit, the meniscus profile can no longer be sustained by the surface tension, and jetting initiates from its tip. The charges induced by the electric field are attracted towards the surface of the drop, and produce an electrostatic polarization stress $p_{e}$ responsible for the early stage deformation of the drop. This polarization stress can be described in the form of a normal electrostatic stress $\sigma_{e}$, related to conduction charges, and a tangential electrostatic stress $\tau_{e}$, related to convection charges ${ }^{31,47}$

$$
\begin{gathered}
\sigma_{e}=\frac{E_{N}^{2}}{2 \varepsilon_{r}}, \\
\tau_{e}=\varepsilon_{0} E_{T} E_{N},
\end{gathered}
$$

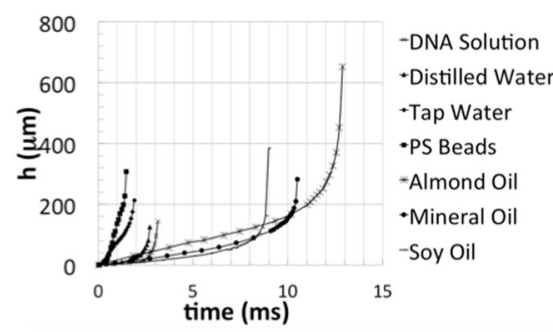

(a)

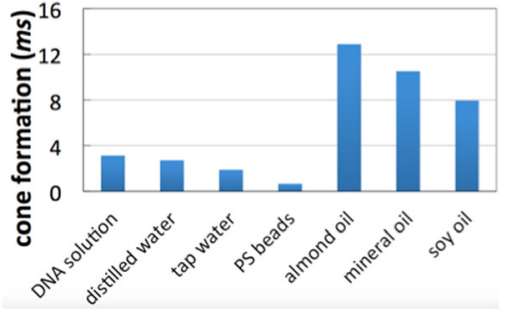

(b)

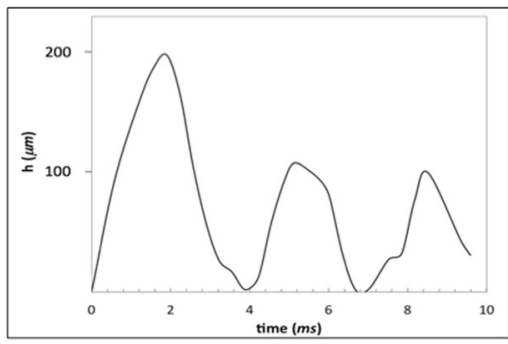

(c)
FIG. 2. (a) Plot of the cone height as a function of time for each liquid; (b) distribution of the time formation of the cone for all of the liquids investigated. (c) Temporal variation of the height of the meniscus apex of the oligonucleotide solution, during the oscillating behaviour. 


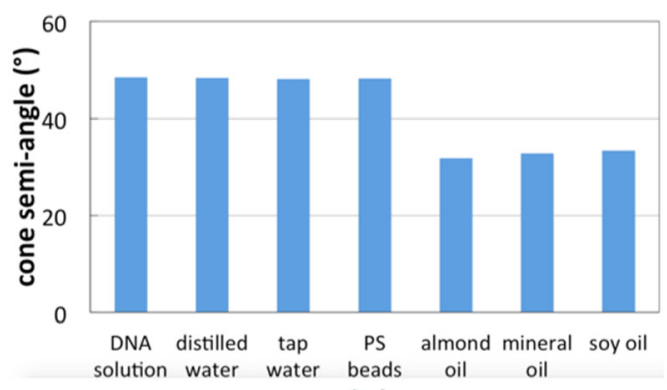

(a)

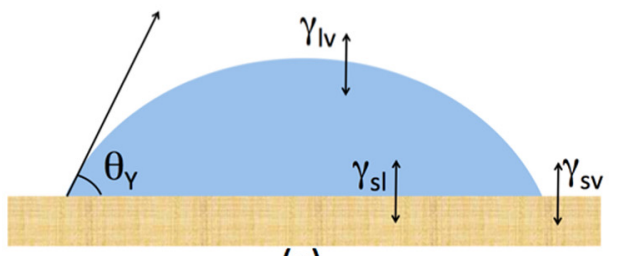

(c)

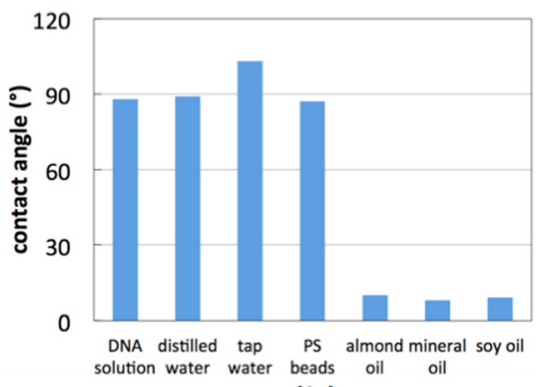

(b)

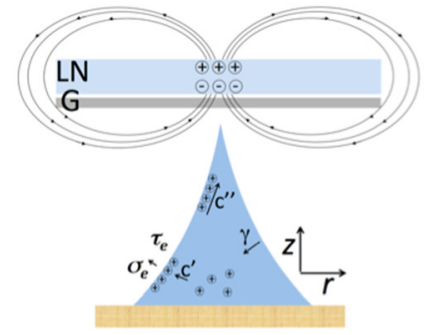

(d)
FIG. 3. Distribution of (a) the cone semi-angle and of (b) the contact angle for each liquid, and schematic view of (c) the base drop under the equilibrium condition and of (d) the base drop in presence of the pyroelectric field. LN: lithium niobate; G: glass slide; $c^{\prime}$ : conduction current; $\mathrm{c}^{\prime \prime}$ : convection current; $\gamma$ : surface tension; $\tau_{e}$ : tangential electric stress; and $\sigma_{e}$ : normal electric stress. where $E_{N}$ is the normal electric field, $\varepsilon_{r}$ is the relative permittivity of the liquid to $\varepsilon_{0}$, and $E_{T}$ is the tangential electric field. The conduction charges move mainly from the bulk towards the surface of the drop and the related normal stress tends to deform the drop into a shape with spherical symmetry. The convection charges are those that migrate along the surface of the liquid, accumulate onto the apex of the drop, ${ }^{47}$ and tend to accelerate the surrounding liquid towards the cone apex, causing the stretching of the meniscus along the interface. At this point, the evolution of the drop deformation depends basically on the velocity of the liquid and of the charges, according to these main parameters: the local strength of the electric field; the permittivity, which is related to the amount of charges in the fluid; the viscosity, which regulates the flow properties of the liquid; the density; the surface tension. In fact, the acceleration of the charges, produced by the electrostatic stresses, makes the fluid to experience viscous stresses, basically the normal viscosity stress $\sigma_{\mu}$ and the tangential viscosity stress $\tau_{\mu}{ }^{47}$

$$
\begin{gathered}
\sigma_{\mu}=2 \mu \frac{\partial V_{z}}{\partial z} \\
\tau_{\mu}=\frac{3 \mu\left(\partial V_{z} / \partial z\right)(d r / d z)}{1+(d r / d z)^{2}}
\end{gathered}
$$

where $\mu$ is the absolute viscosity of the liquid and $V_{z}$ is the liquid velocity in the axial direction. Summarizing, the electric field generates normal and tangential electrostatic stresses that consequently induce normal and tangential viscosity stresses, and the interplay of charge and fluid motions (related to dielectric and fluidic properties of the liquid) forms the jet and regulates its temporal and shape characteristics.

Therefore, the drop evolution differentiates significantly for conductive and dielectric liquids, according to the studies performed in Ref. 38 and in agreement with the experimental results. In case of conductive fluids, the dominant charge transport is due to ohmic conduction and the charging time $t_{C} \propto \varepsilon / \sigma$ ( $\varepsilon$ dielectric constant, $\sigma$ conductivity $)$, which depends basically on the conductivity of the fluid, ${ }^{36}$ is always the shortest timescale in the system. In other words, as the meniscus deforms, due to the charge migration and the related fluid acceleration, those charges migrate to the free surface instantaneously, compared with the fluid motion, thus screening immediately the external electric field. Therefore, the electric field components $E_{T}$ and $E_{N}$ inside the drop vanish for any time and, consequently, the electrostatic stresses $\sigma_{\varepsilon}$ and $\tau_{\varepsilon}$, tend to disappear (see formulas 1 and 2). In this way the force that drives the spherical symmetry of the flow is preserved. As a consequence, the Taylor cone, after sharpening in the typical conic cusping form, cannot go further and returns to the equilibrium state for refilling its charge, in perfect agreement with the result in the supplementary movie 1.45

Conversely, in case of DLs, the local timescale for flow becomes smaller with respect to the charging time and the dominant mode of charge transport is due to convection. The electrostatic stresses $\sigma_{\varepsilon}$ and $\tau_{\varepsilon}$ increase due to a lack of fast compensation of the external electric field at the interface of the liquid. Therefore, the tip of the conical meniscus sharpens, the fluid in the tip accelerates continuously and the inertia of the fluid make the viscosity stresses $\sigma_{\mu}$ and $\tau_{\mu}$ to increase, thus destroying the spherical symmetry of the flow. The cone assumes the typical elongated shape and the inception of tip streaming takes more time and lasts longer (see supplementary movie $2^{45}$ ). These are the reasons that decrease the pulsation frequency of the cone in case of lower conductivities. The different regimes highlighted here for conductive and dielectric liquids are of fundamental importance for all of those applications where precise liquid handling and/or printing is required: in biochemistry, where mainly conductive liquids are manipulated, or in the field of biocompatible scaffolds, where mainly dielectric liquids are employed. The CLs tend to release small droplets at every discharge event exhibiting a pulsating behaviour, while the DLs tend to deform slowly under the action of the electric field and release small droplets while maintaining the cone profile. 
In conclusion, we reported the experimental investigation of the behaviour of sessile drops, with low and high conductivities, under the action of a pyroelectric field. The results show that the CLs exhibited a typical pulsating behaviour, while steady cone behaviour was observed in case of the DLs. The cone formation was characterized in terms of shape and temporal evolution of the meniscus profile, and the results were in good agreement with the predictions reported in literature in case of standard EHD techniques. ${ }^{38}$ Nowadays, we employed the pyro-EHD printing system only for DLs. This close investigation of the drop dynamics demonstrates that the system can be used also for CLs and that the theoretical interpretation well established in case of standard EHD can be adopted also in case of pyroEHD, thus extending its application to all of those fields where conductive liquids are used, such as in biochemistry and in biomedicine.

The authors acknowledge the Italian Ministry of Research for financial support under "Futuro in Ricerca 2010" Programme (Protocol RBFR10FKZH) and under the "Progetto Operativo Nazionale" AquaSystem (Protocol 1719).

${ }^{1}$ R. L. Hines, J. Appl. Phys. 37, 2730 (1966).

${ }^{2}$ J.-U. Park, M. Hardy, S. J. Kang, K. Barton, K. Adair, D. K. Mukhopadhyay, C. Y. Lee, M. S. Strano, A. G. Alleyne, J. G. Georgiadis, P. M. Ferreira, and J. A. Rogers, Nat. Mater. 6, 782 (2007).

${ }^{3}$ D.-Y. Lee, Y.-S. Shin, S.-E. Park, T.-U. Yu, and J. Hwang, Appl. Phys. Lett. 90, 081905 (2007).

${ }^{4}$ J.-U. Park, S. Lee, S. Unarunotai, Y. Sun, S. Dunham, T. Song, P. M. Ferreira, A. G. Alleyene, U. Paik, and J. A. Rogers, Nano Lett. 10, 584 (2010).

${ }^{5}$ C. Wei, H. Qin, N. A. Ramírez-Iglesias, C.-P. Chiu, Y. Lee, and J. Dong, J. Micromech. Microeng. 24, 045010 (2014).

${ }^{6}$ O. A. Basaran, H. Gao, and P. P. Bhat, Annu. Rev. Fluid Mech. 45, 85 (2013).

${ }^{7}$ J.-H. Kim, D.-Y. Lee, J. Hwang, and H.-I. Jung, Microfluid. Nanofluid. 7, 829 (2009).

${ }^{8}$ S. N. Jayasinghe and A. Townsend-Nicholson, Lab Chip 6, 1086 (2006).

${ }^{9}$ S. N. Jayasinghe, A. N. Qureshi, and P. A. M. Eagles, Small 2, 216 (2006).

${ }^{10}$ I. I. Inculet, J. Electrost. 16, 287 (1985).

${ }^{11}$ D.-Y. Chang, C.-C. Lee, and J. Shiea, Anal. Chem. 74, 2465 (2002).

${ }^{12}$ K. L. Choy and B. Su, Thin Solid Films 388, 9 (2001).

${ }^{13}$ J. D. Sartor, Phys. Today 22(8), 45 (1969).

${ }^{14}$ S. L. Cohen, F. Plasil, and W. J. Swiatecki, Ann. Phys. (N.Y.) 82, 557 (1974).
${ }^{15}$ G. Taylor, Proc. R. Soc. A 280, 383 (1964).

${ }^{16}$ G. I. Taylor and A. D. McEwan, J. Fluid Mech. 22, 1 (2006).

${ }^{17}$ F. K. Wohlhuter and O. A. Basaran, J. Fluid Mech. 235, 481 (1992).

${ }^{18}$ M. T. Harris and O. A. Basaran, J. Colloid Interface Sci. 161, 389 (1993).

${ }^{19}$ X. Zhang and O. A. Basaran, J. Fluid Mech. 326, 239 (1996).

${ }^{20}$ P. K. Notz and O. A. Basaran, J. Colloid Interface Sci. 213, 218 (1999).

${ }^{21}$ I. Hayati, A. I. Bailey, and T. F. Tadros, J. Colloid Interface Sci. 117, 205 (1987).

${ }^{22}$ S. Maheshwari and H.-C. Chang, J. Appl. Phys. 102, 034902 (2007).

${ }^{23}$ R. T. Collins, K. Sambath, M. T. Harris, and O. A. Basaran, Proc. Natl. Acad. Sci. U.S.A. 110, 4905 (2013).

${ }^{24}$ A. Lee, H. Jin, H.-W. Dang, K.-H. Choi, and K. H. Ahn, Langmuir 29, 13630 (2013).

${ }^{25}$ H. Kim, J. Song, J. Chung, and D. Hong, J. Appl. Phys. 108, 102804 (2010).

${ }^{26}$ T. K. Nguyen, V. D. Nguyen, B. Seong, N. Hoang, J. Park, and D. Byun, J. Aerosol Sci. 71, 29 (2014).

${ }^{27}$ S. N. Jayasinghe and M. J. Edirisinghe, J. Aerosol Sci. 33, 1379 (2002).

${ }^{28}$ O. Ghazian, K. Adamiak, and G. S. P. Castle, J. Electrost. 71, 489 (2013).

${ }^{29}$ C. Ferrera, J. M. López-Herrera, M. A. Herrada, J. M. Montanero, and A. J. Acero, Phys. Fluids 25, 012104 (2013).

${ }^{30}$ S. B. Q. Tran, D. Byun, V. D. Nguyen, and T. S. Kang, Phys. Rev. E 80, 026318 (2009).

${ }^{31}$ J. L. Li, J. Electrost. 64, 44 (2006).

${ }^{32}$ S. B. Q. Tran and D. Byun, J. Electrost. 71, 28 (2013).

${ }^{33}$ L. L. F. Agostinho, C. U. Yurteri, J. Wartena, S. P. Brouwer, E. C. Fuchs, and J. C. M. Marijnissen, Appl. Phys. Lett. 102, 194103 (2013).

${ }^{34}$ B. Sadri, P. Tabatabaee-Hosseini, B. V. Hokmabad, M. R. Charan, and E. Esmaeilzadeh, J. Colloid Interface Sci. 400, 104 (2013).

${ }^{35}$ W. Wei, Z. Gu, S. Wang, Y. Zhang, K. Lei, and K. Kase, J. Micromech. Microeng. 23, 015004 (2013).

${ }^{36}$ F. Liu and C.-H. Chen, J. Electrost. 72, 330 (2014).

${ }^{37}$ S. N. Reznik, A. L. Yarin, A. Theron, and E. Zussman, J. Fluid Mech. 516, 349 (2004).

${ }^{38}$ R. T. Collins, J. J. Jones, M. T. Harris, and O. A. Basaran, Nat. Phys. 4, 149 (2008).

${ }^{39}$ P. Ferraro, S. Coppola, S. Grilli, M. Paturzo, and V. Vespini, Nat. Nanotechnol. 5, 429 (2010).

${ }^{40}$ S. Grilli, S. Coppola, V. Vespini, F. Merola, A. Finizio, and P. Ferraro, Proc. Natl. Acad. Sci. U.S.A. 108, 15106 (2011).

${ }^{41}$ S. Coppola, V. Vespini, S. Grilli, and P. Ferraro, Lab Chip 11, 3294 (2011).

${ }^{42}$ R. Vecchione, S. Coppola, E. Esposito, C. Casale, V. Vespini, S. Grilli, P. Ferraro, and P. A. Netti, Adv. Funct. Mater. 24, 3515 (2014).

${ }^{43}$ S. Coppola, V. Vespini, G. Nasti, O. Gennari, S. Grilli, M. Ventre, M. Iannone, P. A. Netti, and P. Ferraro, Chem. Mater. 26, 3357 (2014).

${ }^{44}$ J. D. Brownridge and S. Raboy, J. Appl. Phys. 86, 640 (1999).

${ }^{45}$ See supplementary material at http://dx.doi.org/10.1063/1.4907005 for observing the evolution of the drop profile in case of conductive and dielectric liquids.

${ }^{46}$ G. Berg, L. E. Lundgaard, and N. Abi-Chebel, Chem. Eng. Process. 49, 1229 (2010).

${ }^{47}$ R. P. A. Hartman, D. J. Brunner, D. M. A. Camelot, J. C. M. Marijnissen, and B. Scarlett, J. Aerosol Sci. 30, 823 (1999). 